\title{
Implication of long-distance regulation of the HOXA cluster in a patient with postaxial polydactyly
}

\author{
Elisabeth M. Lodder • Bert H. Eussen - Daniëlla A. C. M. van Hassel • \\ A. Jeannette M. Hoogeboom • Pino J. Poddighe • J. Henk Coert • Ben A. Oostra • \\ Annelies de Klein • Esther de Graaff
}

Received: 29 April 2009 / Accepted: 10 June 2009/Published online: 12 August 2009

(C) The Author(s) 2009. This article is published with open access at Springerlink.com

\begin{abstract}
Apparently balanced chromosomal inversions may lead to disruption of developmentally important genes at the breakpoints of the inversion, causing congenital malformations. Characterization of such inversions may therefore lead to new insights in human development. Here, we report on a de novo inversion of chromosome 7 (p15.2q36.3) in a patient with postaxial polysyndactyly. The breakpoints do not disrupt likely candidate genes for the limb phenotype observed in the patient. However, on the p-arm the breakpoint separates the HOXA cluster from a gene desert containing several conserved noncoding elements, suggesting that a disruption of a cis-regulatory circuit of the HOXA cluster could be the underlying cause of the phenotype in this patient.
\end{abstract}

Keywords pericentric chromosomal inversion . postaxial polysyndactyly · congenital malformation . chromosome $7 \cdot$ HOXA cluster

Responsible Editor: Herbert Macgregor.

E. M. Lodder • B. H. Eussen - D. A. C. M. van Hassel •

A. J. M. Hoogeboom - P. J. Poddighe - B. A. Oostra

A. de Klein · E. de Graaff $(\bowtie)$

Department of Clinical Genetics, Erasmus MC, PO Box 2040, 3000 CA Rotterdam, The Netherlands e-mail: e.degraaff@erasmusmc.nl

J. H. Coert

Department of Plastic Surgery, Erasmus MC,

Rotterdam, The Netherlands

\author{
Abbreviations \\ BAC bacterial artificial chromosome \\ FISH fluorescence in-situ hybridization \\ GLI3 GLI-Kruppel family member 3 \\ NFE2L3 nuclear factor (erythroid-derived 2)-like 3 \\ PCR polymerase chain reaction \\ SD standard deviation \\ SHH sonic hedgehog \\ SNP single nucleotide polymorphism \\ UBE3C ubiquitin protein ligase E3C
}

\section{Introduction}

Pericentric inversions are relatively common chromosomal abnormalities with an incidence $0.012 \%$ in newborns (Morel et al. 2007). These are generally without consequence for the carrier (Morel et al. 2007). However, de novo chromosomal inversions in patients with congenital malformations can be the causative factor of the malformation through disruption of developmentally important genes at the breakpoints (Lybaek et al. 2008).

Postaxial polydactyly (OMIM \#174200) is a congenital malformation in which additional digits are present on the posterior (little finger) side of the hand and or feet. Two phenotypic varieties have been described; in type A the extra digit is well formed and articulates with the fifth or an extra metacarpal. In type B the extra digit is not well 
formed and is frequently in the form of a skin tag (Winter and Tickle 1993). Syndactyly of the additional digits and the most posterior digits is often observed (Castilla et al. 1998). Here, we describe a patient with postaxial polysyndactyly type A carrying an inversion on chromosome 7. Chromosomal abnormalities of human chromosome 7 are mapped and annotated in order to get a better understanding of the functioning of entire chromosomes (Scherer et al. 2003). The chromosome 7 database (http://www.chr7.org) lists 23 chromosomal aberrations that are associated with polydactyly. Seven of these aberrations are related to two genes that are important in limb development: GLI-Kruppel family member 3 (GLI3) on the p-arm and sonic hedgehog (SHH) on the q-arm. Both genes lie in the vicinity of the suspected breakpoints of the inversion in our patient; this prompted us to investigate the inversion in more detail.

We carefully mapped the breakpoints on both arms of chromosome 7, using fluorescence in-situ hybridization followed by Southern blot analysis. We cloned the chromosomal breakpoints by polymerase chain reaction and direct sequencing. Neither breakpoint disrupts a gene that is likely to be involved in limb development. On the q-arm the inversion breakpoint lies in an intergenic nonconserved region. On the p-arm the inversion disrupts NFE2L3, a member of the erythroid cap'n'collar transcription factors (Kobayashi et al. 1999). However, this breakpoint is located $1 \mathrm{Mb}$ telomeric of the HOXA gene cluster, resulting in a separation of the HOXA cluster and a gene desert harboring several conserved noncoding elements. This suggests that a disruption of a cis-regulatory circuit of the HOXA cluster could be the underlying cause of the phenotype in this patient.

\section{Materials and methods}

Case and family history

The patient was born at 37 weeks gestational age after an uneventful pregnancy as the first child of nonconsanguineous parents, without a family history of congenital malformations. His birth weight was $2630 \mathrm{~g}(-1.5 \mathrm{SD}$ for the average population). The boy presented with postaxial polydactyly type A and syndactyly 3-6 of both hands and feet. Autoradiography showed a shortened ulna and overgrown radial head on both sides. An extension limitation of the left knee was observed, due to a congenital flexioncontraction $\left(98^{\circ}\right)$ with webbing of the fossa poplitea. The left knee also presented with a lateral patella luxation. On examination at 19 months his length was $79 \mathrm{~cm}(-2 \mathrm{SD})$ and his weight was $8.4 \mathrm{~kg}(<-2$ SD weight-length ratio). At 4 years 9 months his length was $96.4 \mathrm{~cm}(-3.5 \mathrm{SD}$ for the population average; $-2.5 \mathrm{SD}$ for his target height) and his weight was $13.8 \mathrm{~kg}$ ( $-1 \mathrm{SD}$ weight-length ratio), and his occipitofrontal circumference was $50.3 \mathrm{~cm}$ (between 0 and -1 SD). No other major or minor congenital malformations were found. His psychomotoric development was normal. Both parents were unaffected. Informed consent was obtained from the patient and his parents.

\section{Karyotyping}

Karyotyping was performed on GTG-banded metaphases obtained from peripheral blood cultures of the patient and his parents, using standard procedures (van den Berg et al. 2000). The results were described in accordance with the ISCN 2005 (Shaffer and Tommerup 2005).

\section{ISH}

Fluorescence in-situ hybridization (FISH) analysis was performed as described before (Eussen et al. 2007). Bacterial artificial chromosomes (BACs) and fosmids were ordered from BACPAC Resource Center at the Children's Hospital Oakland Research Institute in Oakland, California (http://bacpac.chori. org/). The relevant BAC clones and fosmids are shown in Fig. 2. Hybridization locations were validated on reference human metaphase spreads.

\section{Affymetrix SNP array}

DNA from the patient was isolated from peripheral blood according to standard procedures and hybridized on the 250K NspI single nucleotide polymorphism array (Affymetrix, Santa Clara, CA, USA) according to the manufacturer's protocols. Hybridization intensities were compared with a well-characterized set of reference individuals. 


\section{Southern blotting}

Southern blotting was performed according to standard protocols (Sambrook et al. 1989). Probes were amplified using radioactive polymerase chain reaction (PCR), using Roche (Mannheim, Germany) Taq polymerase in $10 \mathrm{mM}$ Tris- $\mathrm{HCl}, \mathrm{pH} 8.3,50 \mathrm{mM}$ $\mathrm{KCl}, 1.5 \mathrm{mM} \mathrm{MgCl} 2,50 \mu$ molar of dCTP, dGTP and dTTP, $5 \mu$ molar dATP, $125 \mu \mathrm{Ci}$ of 32P-labeled dATP (Applied Biosystems, Nieuwekerk a.d. IJssel, The Netherlands) and the following primer combinations: Q40: fw 5'-cttctaatgcctgacaatgagg-3', rev 5'-ggccagcgggtctaacctc-3', 1569 bp; P15: fw 5'ggcttcctgtagtgtgaattttg $3^{\prime}$, rev 5'-gaactcttagacacaatatcc3', 1001 bp; P20: fw 5'-ggtgtcagcccagaaggaga-3', rev 5'-ccagggctaccatgatgacagc-3', 444 bp. PCR cycling conditions were $5 \min 94^{\circ} \mathrm{C} ; 32$ cycles of $30 \mathrm{~s} 94^{\circ} \mathrm{C}$, $30 \mathrm{~s} 60^{\circ} \mathrm{C}, 1 \min 30 \mathrm{~s} 72^{\circ} \mathrm{C}$, and 1 cycle of $5 \min 72^{\circ} \mathrm{C}$. Radioactive blots were scanned using a phosphor imager and a Typhoon 9410 workstation (GE Healthcare, Zeist, The Netherlands).

PCR and sequencing of the breakpoints

To sequence the breakpoints of the inversion we designed primers in the vicinity of the suspected breakpoints and deletions for PCR amplification. PCR conditions were as described above (replacing the 32P-labeled dATP with $45 \mu$ molar unlabeled dATP). The resulting PCR products were sequenced using Big Dye Terminator 3.1 (Applied Biosystems). Fragments were loaded on an ABI3100 sequence analyzer and analyzed with DNA Sequencing Analysis (ver.3.7) and SeqScape (ver.2.1) software (Applied Biosystems). Primer sequences were: 7 p-breakpoint: fw 5'-gggctggtttcttcagggcacag-3', rev 5'-tgaaaatcc cactgt gaacgc-3', 529 bp; 7q-breakpoint: fw 5'cgcacaaaccaggtggaagcc-3', rev 5'-ctcattgtcaggcatta gaagc-3', $767 \mathrm{bp} ; \Delta 200$ : fw 5'-gcacatcttgcaccgccc-3', rev 5'-caacctccacctccagctg-3', 1166 bp; $\Delta 773$ : fw 5'-ccataagacagagtactgaggg-3' rev 5'-taagtcttccagaga gaaagaag-3', 1496 bp.

Real-time PCR cDNA analysis

RNA was isolated from Epstein-Barr virus (EBV)transformed lymphoblastoid cell lines (Bliek et al. 2004) of the patient and healthy controls using RNAbee (BioConnect, Huissen, the Netherlands) according to the manufacturer's protocol. Singlestrand cDNA synthesis was performed with SuperScript III (Invitrogen, Breda, the Netherlands). Relative expression levels were measured using iQ SYBR Green Supermix (Biorad, Veenendaal, The Netherlands) on a ABI7300 Real time Cycler (Applied Biosystems) with the following cycling conditions: $2 \min 50.0^{\circ} \mathrm{C}, 10 \min 95.0^{\circ} \mathrm{C} ; 40$ cycles of first $15 \mathrm{~min} 95.0^{\circ} \mathrm{C}$ followed by $1 \mathrm{~min} 60.0^{\circ} \mathrm{C}$; data were acquired during the $60.0^{\circ} \mathrm{C}$ step. Primers were designed with the aid of PrimerExpress 1.0 (Applied Biosystems) software in such a way that forward and reverse primer were located in different exons, to exclude influences of genomic DNA contamination on the real-time results. All PCR products are between 95 and 105 bp long. Primer sequences were: NFE2L3: fw1 5'-aaggcacccgcggaac-3', rev1 5'-atcca cagcttcgtgctttc-3'; fw2 5'-atgagagacatctgaatgggacag3', rev2 5'-agatgccetccagtgaatttc-3'; UBE3C: fw 5'gaagaaaggcgaaggttgaaaaa-3', rev 5'-tgcacttctttggatg gaatattg-3'; HNRNPA2B1: fw 5'-gcttaagctttgaaacca cagaaga-3', rev 5'-tgettgcaggatcc ctcatta-3'; HOXA7: fw 5'-cccgcttcctgtcaggtc-3', rev 5'-gctctgcagtgacctcgc3'; HOXA11: fo 5'-cgtc ttccggccacactga-3', rev 5'ctagctcceggatctggt act-3'; HOXA13: fw 5'-caaatg tactgccccaaagagc-3', rev 5'-ataggagctggcatccgagg-3'; SHH: fw 5'- atgaa gaaaacaccggagcg-3', rev 5'-tcat caccgagatggccaaag- $3^{\prime}$.

\section{Results}

The karyotype of the patient revealed two abnormalities: a duplication of the $\mathrm{Y}$ chromosome and an inversion on chromosome 7 (47, XYY, inv(7) (p15q36). Karyotyping of the parents revealed no abnormalities; the inversion of chromosome 7 is therefore de novo. The 47, XYY genotype is common (incidence 1:1000-1:500 in newborn males) and is not associated with any overt adverse phenotypes (Rives et al. 2003). Therefore, the de novo inversion of chromosome 7 (Fig. 1a) most likely causes the polysyndactyly seen in the patient. We aimed to determine the precise breakpoints of the inversion at DNA level. Different techniques were used, narrowing down the breakpoint areas with each step.

On the basis of the karyotype (Fig. 1a) we mapped the breakpoints using FISH. Several BACs were tested on $7 \mathrm{p} 15$ and $7 \mathrm{q} 36$ (data not shown). The 
a

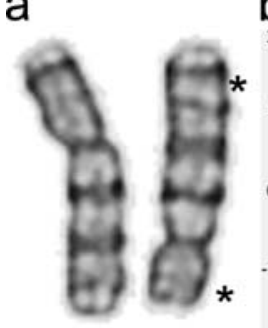

$7 \operatorname{lnv}(7)$ $b_{30}$

3.0

1.5

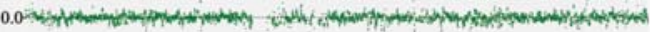

$-1.5$

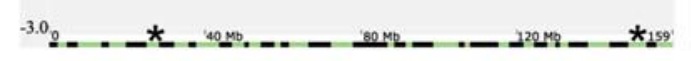

C

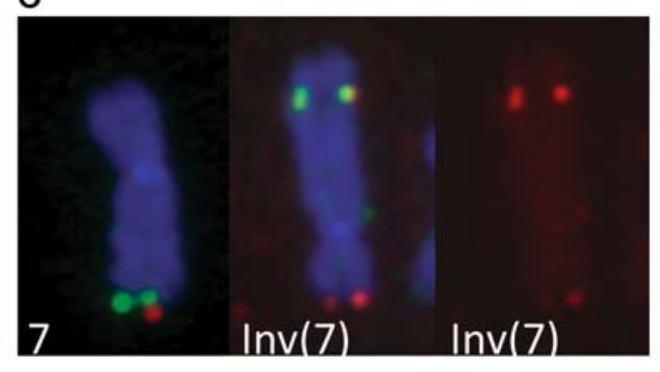

ApaLI

XbaLI

HindIII

EcoRV
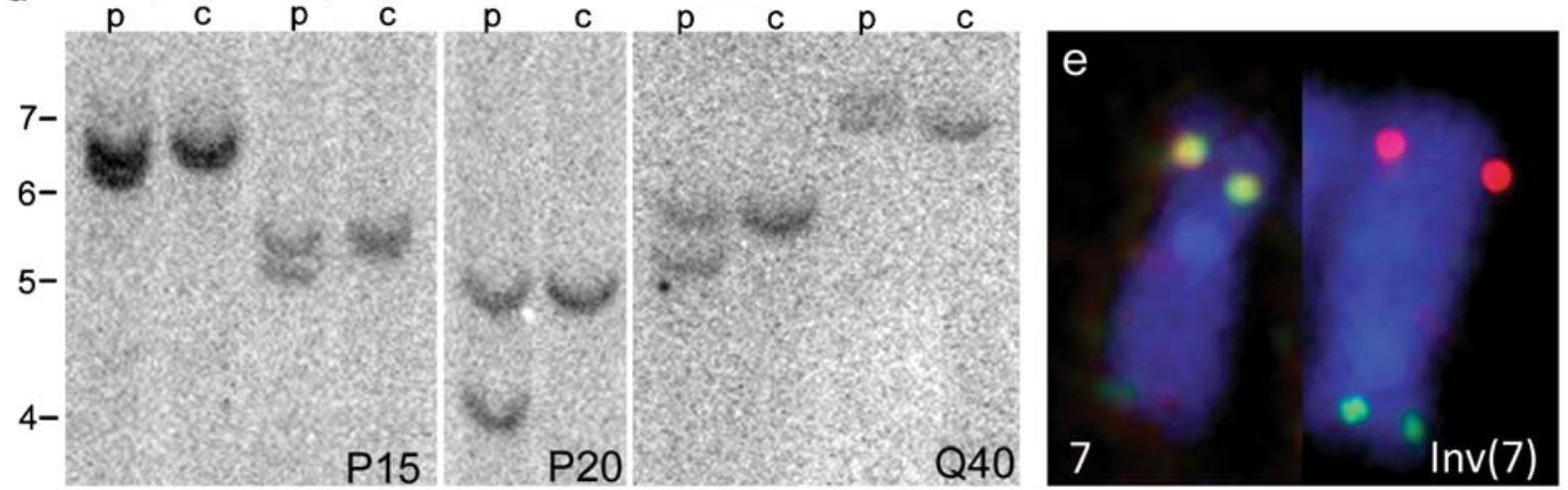

f

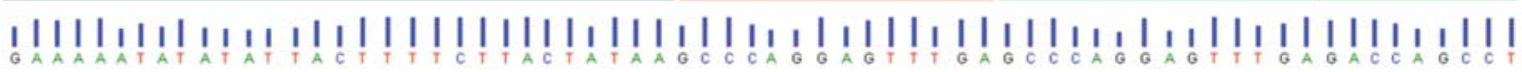

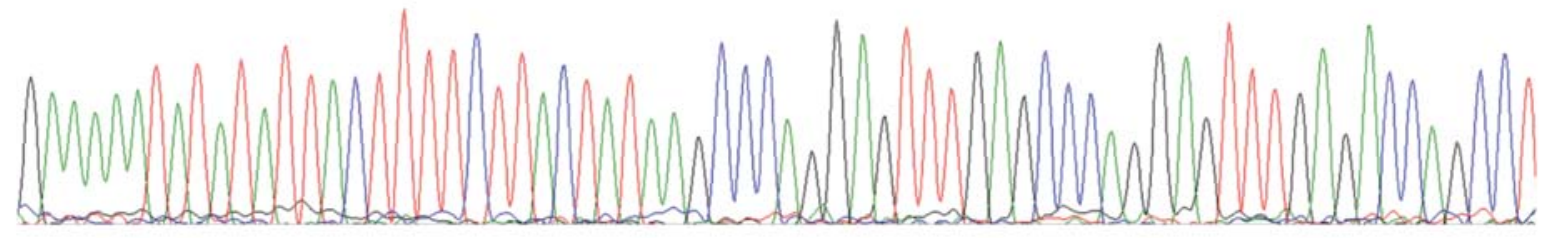

g
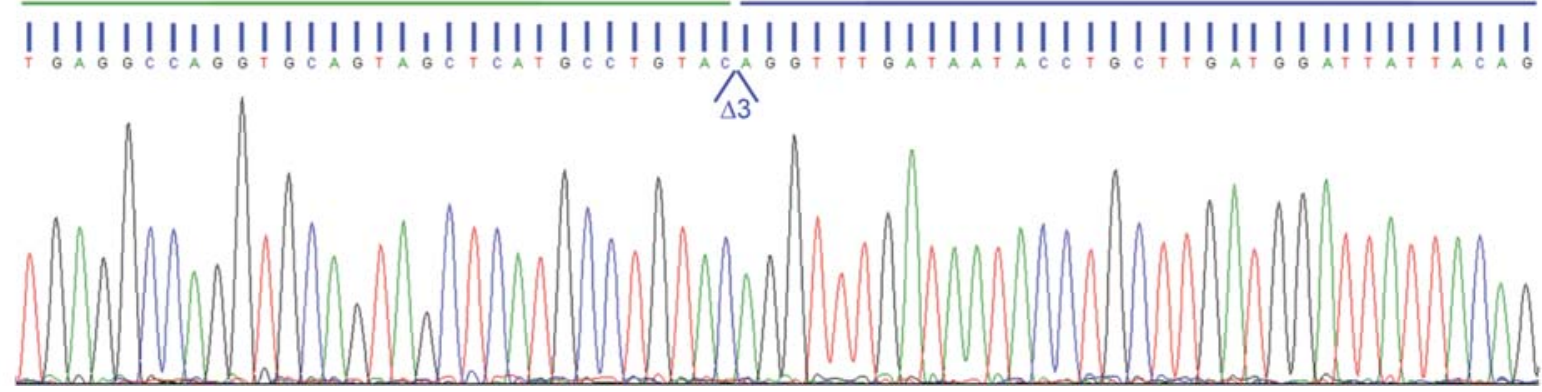

Fig. 1 Detailed mapping of the inversion breakpoints (a) CTG banding of chromosome 7 breakpoints of the inversion are indicated by stars. (b) Affymetrix SNP array results for chromosome 7, revealing no large deletions or duplications; breakpoints of the inversion are indicated by stars, (c) FISH analysis of the breakpoint on $7 \mathrm{q}$ showing G248P85855B2 (green signal swaps to 7p) and G248P89016G3 (red, split signal). (d) Southern blot analysis of probe P15, P20, and Q40 showing abnormal sized bands in the patient (p) and not in the control (c). (e) FISH analysis of the breakpoint on $7 p$ showing separation of fosmids G248P81811A6 (red stays on p) and G248P89547A12 (green, swaps to q). (f, g) Sequence analysis of the breakpoints $7 \mathrm{p}(\mathrm{f}, \mathrm{rev})$ and $7 \mathrm{q}(\mathrm{g}, \mathrm{rev})$. Green, blue, and red lines indicate sequence from $7 \mathrm{p}, 7 \mathrm{q}$, and duplicated sequence from $7 \mathrm{p}$ respectively 
breakpoints were first mapped to the regions covered by BACs RP11-66O14 on the p-arm and RP11-51L24 on the q-arm (data not shown). The breakpoints were further fine-mapped with fosmids covering the BACs. On the short arm of chromosome 7 the breakpoint was mapped to the region between fosmid G248P81511A6, which stayed on the p-arm, and fosmid G248P89547A12, which showed a signal on the q-arm of the inverted chromosome (Fig. 1e). The breakpoint on the q-arm was mapped to fosmid G248P89016G3, which gave a split signal on the inverted chromosome 7 (Fig. 1c). To exclude large genomic imbalances at the inversion breakpoints we performed a SNP array on DNA from the patient. The average probe spacing of the array is $10 \mathrm{~kb}$ at $7 \mathrm{p} 15.2$ and $15 \mathrm{~kb}$ at $7 \mathrm{q} 36.3$. No deletions or duplications were found (Fig. 1b).

Within the regions on both arms indicated by the fosmids we designed regularly spaced probes for Southern blot analysis. On the q-arm probe Q40 showed different-sized bands for 7 different restriction enzymes. On the basis of these restriction fragments the breakpoint on $\mathrm{q}$ was mapped to a 200 bp region (Fig. 1d and data not shown). Furthermore, the size of the aberrant fragments from the inverted chromosome 7 was used to determine the position of the breakpoint on the p-arm. On the p-arm probes P15 and P20 showed double bands with multiple restriction enzymes (Fig. 1d). The difference between the abnormal and the normal band size for probe P15 and P20 was the same for all restriction enzymes ( $\sim 200$ bp and $\sim 800$ bp respectively), indicating the presence of small heterozygous deletions in the patient.

Next, we designed PCR primers around the two deleted regions and both breakpoints to determine their exact locations. On the q-arm, the breakpoint lies $20 \mathrm{~kb}$ before the transcriptional start site of ubiquitin protein ligase $\mathrm{E} 3 \mathrm{C}$ (UBE3C) in a nonconserved region (Fig. 2). UBE3C is a 'homologous to E6AP C-terminus' family member involved in substrate protein selection for ubiquitin ligation (Wang et al. 2006).

The deletion detected by probe P15 is a complex rearrangement of a highly repetitive region in intron 1 of NFE2L3, partially covering variation_28613 (Levy et al. 2007) resulting in the deletion of approximately $200 \mathrm{bp}$. The deletion detected by P20 is a 773 bp deletion in intron 2 of NFE2L3. See Fig. 2 for an overview of the positions of the deletions in NFE2L3.
Both deletions are not de novo as the father carries the $\Delta 773$ deletion and the mother the $\Delta 200$ deletion (data not shown). Moreover, both deletions involve highly repetitive intronic regions, indicating that it is highly unlikely that either deletion causes the polysyndactyly.

The breakpoint on the p-arm of chromosome 7 lies within intron 1 of NFE2L3. The inversion of chromosome 7 in this patient leads therefore to the disruption of NFE2L3 (Fig. 2). The sequences of the breakpoints revealed that the inversion is not completely balanced. On the p-arm a 14-nucleotide duplication of a repeat sequence was detected. On $7 \mathrm{q}$ the inversion has resulted in the deletion of 3 nucleotides (Fig $1 \mathrm{f}-\mathrm{g}$ ). It is unclear whether a relationship exists with either of the small deletions. The distance between the breakpoint and the deletions is too large to be able to determine whether the deletion is on the paternal or the maternal chromosome.

We tested the expression levels of the genes surrounding the breakpoints (NFE2L3, HNRNPA2/ $\mathrm{B} 1$, and $\mathrm{UBE} 3 \mathrm{C}$ ) and of candidate genes in the vicinity (sonic hedgehog (SHH) and genes in the HOXA cluster) in EBV-transformed cell lines of the patient and controls. No changes in expression level were detected for UBE3C, HNRNPA2B1, and SHH and the expression level of NFE2L3 was reduced to $50 \%$ as expected (data not shown). The level of expression of HOXA7, HOXA11, and HOXA13 was too variable in all (control and patient) EBV cell lines to allow conclusions to be drawn (data not shown).

\section{Discussion}

We determined the exact positions of the breakpoints of a chromosome 7 inversion in a patient with polysyndactyly. These breakpoints disrupt only one gene: NFE2L3. NFE2L3 is a member of the cap'n'collar erythroid transcription factor family (Kobayashi et al. 1999) and is suggested to have a role in the oxidative stress response (Sankaranarayanan and Jaiswal 2004; Chowdhury et al. 2009). During development, NFE2L3 is expressed in mesodermal derivatives in avian embryos; no expression of NFE2L3 is detected in the limb buds (Etchevers 2005). Mice homozygous for a knockout allele of NFE2L3 (deleting exon 3 and 4) lack a limb phenotype (Derjuga et al. 2004). Therefore, the disruption of the NFE2L3 gene 


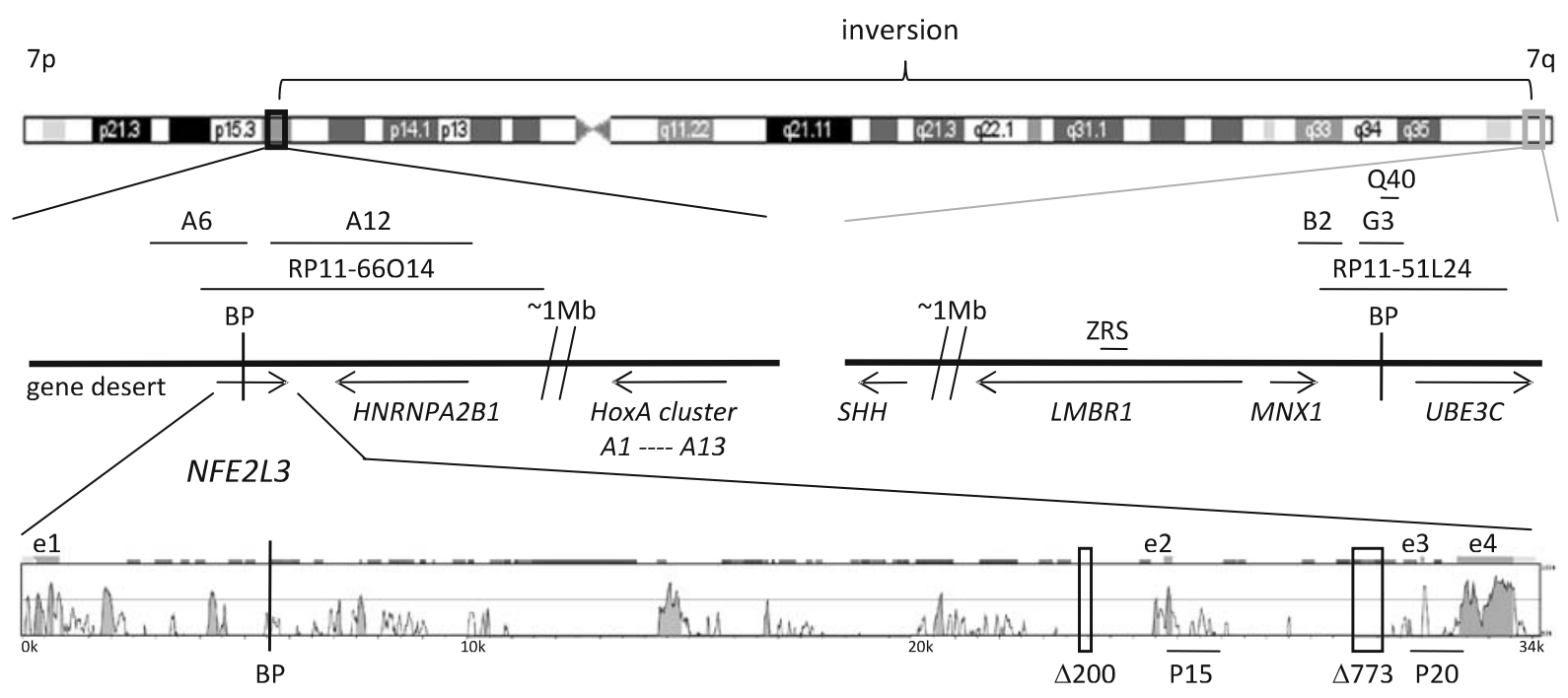

Fig. 2 Overview of the inversion on chromosome 7: From top to bottom: karyogram of chromosome 7 (based on the Ensembl genome browser http://www.ensembl.org/). Black rectangle: area of the breakpoint on $7 \mathrm{p}$; gray rectangle: area of the breakpoint on 7q. Locations of the BACs (RP11-66O14 and RP11-51L24) and fosmids A6 (G248P81811A6), A12 (G248P89547A12), B2 (G248P85855B2), and G3 (G248P89016G3), and probe Q40 are indicated by lines. Genomic context (not to scale) of the breakpoints (BP), indicating the position of genes flanking the

by the inversion and the two inherited small intronic deletions are very unlikely to cause the limb phenotype observed in the patient.

It is possible that the phenotype is caused by a de novo point mutation, coinciding with the observed inversion. We therefore also studied the most likely gene involved in postaxial polydactyly, GLI3. Sequencing of the coding regions of this gene did not reveal any mutation, however, other than known polymorphisms (data not shown).

As the phenotype could not be explained by the disruption of NFE2L3, we hypothesized that disruption of a cis-regulatory circuit of a developmentally important gene could cause the phenotype. On the q-arm the breakpoint is located $1.3 \mathrm{Mb}$ upstream from $\mathrm{SHH}$. Although it would be possible that the breakpoint separates SHH from a second long-distance regulatory element, this option is not very likely considering the phenotype. Thus far, SHH mutations have always been associated with either holoprosencephaly or preaxial polydactyly. Furthermore, the synteny between the human chromosome 7 and the mouse chromosome 5 breaks just centromeric of the breakpoint, limiting the region that could contain the possible enhancer. No breakpoints and those of particular interest due to their role in limb development (ZRS: limb specific SHH cis-regulatory element). Homology plot of NFE2L3 (http://www.gSD.lbl.gov/ vista); stretches of homology $>75 \%$ between human and mouse are indicated in gray. The line marks the position of the breakpoint, the rectangles indicate the deletions, e1-4 mark the positions of the exons, and probes $\mathrm{P} 15$ and $\mathrm{P} 20$ are indicated by lines

likely candidate conserved elements are obvious in this region.

The p-breakpoint of the inversion lies between a gene desert containing several multispecies conserved noncoding elements (Fig. 2) and the HOXA cluster. The homeobox transcription factors of the HOXA and HOXD cluster are known to play important roles during development. One of their functions is the patterning of the anterior to posterior axis of the limbs. Correct timing and position of expression is essential for the correct activation of downstream targets such as $\mathrm{SHH}$, fibroblast growth factors, and bone morphogenetic proteins (Zakany and Duboule 2007). Slight misregulation of the expression of the HOX genes, as a result of heterozygous mutations, can cause limb malformations, such as polydactyly (Goodman 2002; Montavon et al. 2008).

On the basis of the location of the breakpoint, the distance between the conserved noncoding elements in the gene desert and the HOXA cluster would range from 1 to $1.8 \mathrm{Mb}$. Although these distances are relatively large, regulatory elements that function over such distances have been described, especially in the context of developmentally specific enhancers (Jamie- 
son et al. 2002; Lettice et al. 2003; Bagheri-Fam et al. 2006; Bien-Willner et al. 2007). Recent work by Duboule and colleagues has shown that the distance between the HOXD cluster and its regulatory elements is dependent upon the time in evolution when the expression pattern was acquired. Elements regulating the basic expression cascade along the trunk are close to the cluster, whereas elements regulating later expression such as in the limb are located farther away (Spitz and Duboule 2008).

How the expression of the HOXA cluster is regulated in the limb is thus far not clear. A recent paper by Lehoczky shows the presence of at least two limb enhancers approximately $300 \mathrm{~kb} \mathrm{5'}$ of the HOXA cluster (Lehoczky and Innis 2008). However, these enhancers are not capable of recapitulating the complete limb expression pattern of the HOXA genes. The inversion on chromosome 7 may suggest the presence of long-distance regulatory elements of the HOXA cluster in the gene desert telomeric of NFE2L3.

In summary, we have mapped the breakpoints of a de novo inversion on chromosome 7 in a patient with postaxial polysyndacytyly. The breakpoints do not seem to disturb developmentally important genes. Therefore, the most likely explanation of the polysyndactyly is the disruption of a cis-regulatory circuit of gene(s) that are involved in limb development. This may indicate the presence of a long-distance regulatory element of the HOXA cluster within a gene desert telomeric of the breakpoint on the short arm of chromosome 7. Future genetic analysis of polydactyly in both mice and humans will be necessary to confirm the existence of such a regulatory element.

Acknowledgements We thank the patient and his family for their cooperation, and Wilfred van IJcken (Erasmus MC Department of Biomics) for microarray hybridizations. E. de Graaff is supported by a grant from the Dutch Scientific Organization (NWO, 016.056.320).

Open Access This article is distributed under the terms of the Creative Commons Attribution Noncommercial License which permits any noncommercial use, distribution, and reproduction in any medium, provided the original author(s) and source are credited.

\section{References}

Bagheri-Fam S, Barrionuevo F, Dohrmann U et al (2006) Longrange upstream and downstream enhancers control distinct subsets of the complex spatiotemporal Sox9 expression pattern. Dev Biol 291:382-397

Bien-Willner GA, Stankiewicz P, Lupski JR (2007) SOX9cre1: a cis-acting regulatory element located $1.1 \mathrm{Mb}$ upstream of SOX9: mediates its enhancement through the $\mathrm{SHH}$ pathway. Hum Mol Genet 16:1143-1156

Bliek JB, de Klein A, Luider TM et al (2004) New approach for the identification of folate-related pathways in human embryogenesis. Cell Mol Biol (Noisy-le-grand 50:939944

Castilla EE, Lugarinho R, da Graca Dutra M, Salgado LJ (1998) Associated anomalies in individuals with polydactyly. Am J Med Genet 80:459-465

Chowdhury I, Mo Y, Gao L, Kazi A, Fisher AB, Feinstein SI (2009) Oxidant stress stimulates expression of the human peroxiredoxin 6 gene by a transcriptional mechanism involving an antioxidant response element. Free Radic Biol Med 46:146-153

Derjuga A, Gourley TS, Holm TM et al (2004) Complexity of CNC transcription factors as revealed by gene targeting of the Nrf3 locus. Mol Cell Biol 24:3286-3294

Etchevers HC (2005) The cap ' $n$ ' collar family member NF-E2related factor $3(\mathrm{Nrf} 3)$ is expressed in mesodermal derivatives of the avian embryo. Int J Dev Biol 49:363-367

Eussen BH, van de Laar I, Douben H et al (2007) A familial inverted duplication 2q33-q34 identified and delineated by multiple cytogenetic techniques. Eur $\mathrm{J}$ Med Genet 50:112-119

Goodman FR (2002) Limb malformations and the human HOX genes. Am J Med Genet 112:256-265

Jamieson RV, Perveen R, Kerr B et al (2002) Domain disruption and mutation of the bZIP transcription factor MAF, associated with cataract, ocular anterior segment dysgenesis and coloboma. Hum Mol Genet 11:33-42

Kobayashi A, Ito E, Toki T et al (1999) Molecular cloning and functional characterization of a new Cap'n' collar family transcription factor Nrf3. J Biol Chem 274:6443-6452

Lehoczky JA, Innis JW (2008) BAC transgenic analysis reveals enhancers sufficient for Hoxa13 and neighborhood gene expression in mouse embryonic distal limbs and genital bud. Evol Dev 10:421-432

Lettice LA, Heaney SJ, Purdie LA et al (2003) A long-range Shh enhancer regulates expression in the developing limb and fin and is associated with preaxial polydactyly. Hum Mol Genet 12:1725-1735

Levy S, Sutton G, Ng PC et al (2007) The diploid genome sequence of an individual human. PLoS Biol 5:e254

Lybaek H, Oyen N, Fauske L, Houge G (2008) A $2.1 \mathrm{Mb}$ deletion adjacent but distal to a $14 \mathrm{q} 21 \mathrm{q} 23$ paracentric inversion in a family with spherocytosis and severe learning difficulties. Clin Genet 74:553-559

Montavon T, Le Garrec JF, Kerszberg M, Duboule D (2008) Modeling Hox gene regulation in digits: reverse collinearity and the molecular origin of thumbness. Genes Dev 22:346-359

Morel F, Laudier B, Guerif F et al (2007) Meiotic segregation analysis in spermatozoa of pericentric inversion carriers using fluorescence in-situ hybridization. Hum Reprod 22:136-141

Rives N, Simeon N, Milazzo JP, Barthelemy C, Mace B (2003) Meiotic segregation of sex chromosomes in mosaic and 
non-mosaic XYY males: case reports and review of the literature. Int J Androl 26:242-249

Sambrook J, Fritsch E, Maniatis T (1989) Molecular Cloning: A Laboratory Manual. Cold Spring Harbor Laboratory Press, Cold Spring Harbor NY

Sankaranarayanan K, Jaiswal AK (2004) Nrf3 negatively regulates antioxidant-response element-mediated expression and antioxidant induction of $\mathrm{NAD}(\mathrm{P}) \mathrm{H}$ : quinone oxidoreductase1 gene. J Biol Chem 279: 50810-50817

Scherer SW, Cheung J, MacDonald JR et al (2003) Human chromosome 7: DNA sequence and biology. Science 300:767-772

Shaffer LG, Tommerup N (eds) (2005) ISCN 2005: An International System for Human Cytogenetic Nomenclature (2005). Basel: S. Karger
Spitz F, Duboule D (2008) Global control regions and regulatory landscapes in vertebrate development and evolution. Adv Genet 61:175-205

van den Berg C, Van Opstal D, Brandenburg H et al (2000) Accuracy of abnormal karyotypes after the analysis of both short- and long-term culture of chorionic villi. Prenat Diagn 20:956-969

Wang M, Cheng D, Peng J, Pickart CM (2006) Molecular determinants of polyubiquitin linkage selection by an HECT ubiquitin ligase. EMBO J 25:1710-1719

Winter RM, Tickle C (1993) Syndactylies and polydactylies: embryological overview and suggested classification. Eur J Hum Genet 1:96-104

Zakany J, Duboule D (2007) The role of Hox genes during vertebrate limb development. Curr Opin Genet Dev 17:359-366 